\title{
Predictive value of preoperative serum CCL2, CCL18, and VEGF for the patients with gastric cancer
}

Jianghong $\mathrm{Wu}^{1,2+}$, Xiaowen Liu ${ }^{1,2+}$ and Yanong Wang ${ }^{1,2^{*}}$

\begin{abstract}
Background: To investigate the expression of chemokine ligand 2 (CCL2), chemokine ligand 18 (CCL18), and vascular endothelial growth factor (VEGF) in peripheral blood of patients with gastric cancer and their correlation with presence of malignancy and disease progression.

Methods: Sixty patients with pathological proved gastric cancer were prospectively included into study. The levels of CCL2, CCL18, and VEGF in peripheral blood were examined by enzyme-linked immunosorbentassay (ELISA). Peripheral blood from 20 healthy people was examined as control.

Results: The preoperative serum levels of CCL2, CCL18 and VEGF in gastric cancer patients were significantly higher than that of controls $(P<0.001, P<0.001$, and $P<0.001$, respectively). ROC curve analysis showed that with a cut-off value of $\geq 1272.8$, the VEGF*CCL2 predicted the presence of gastric cancer with $83 \%$ sensitivity and $80 \%$ specificity. Preoperative serum CCL2 was significantly correlated to $N$ stage $(P=0.040)$; CCL18 associated with $N$ stage $(P=0.002)$, and TNM stage $(P=0.002)$; VEGF correlated to T stage $(P=0.000)$, N stage $(P=0.015)$, and TNM stage $(P=0.000)$.
\end{abstract}

Conclusion: Preoperative serum levels of CCL2 and VEGF could play a crucial role in predicting the presence and progression of gastric cancer.

Keywords: Gastric cancer, Diagnosis, Biological markers

\section{Background}

Although the incidence of gastric cancer has been substantially declining for several decades, it was still the fourth most common cancer and the second most frequent cause of cancer death [1,2]. The high death rate was related to the difficulty of detecting gastric cancer at an early stage. Some serum tumor markers such as AFP, CEA, CA19-9, CA72-4, and CA50 were currently the best clinical markers for gastric cancer. However, they were no good diagnostic tumor makers in early stage gastric cancer. Therefore, it was important to develop new markers for detecting gastric cancer.

\footnotetext{
* Correspondence: wangyn1111@hotmail.com

${ }^{\dagger}$ Equal contributors

${ }^{1}$ Department of Gastric Cancer and Soft Tissue Sarcoma, Fudan University Shanghai Cancer Center, 270 Dong An Road, Shanghai 200032, People's Republic of China

Department of Oncology, Shanghai Medical College, Fudan University, Shanghai 200032, China
}

Chemokines were a kind of low-molecular weight cytokines, which were implicated in many biological processes, such as migration of leukocytes, angiogenesis, and tumor growth. The CCL chemokines represented the largest family of chemokines. They could attract monocytes, macrophages, T cells, B cells, eosinophils, dendritic cells, mast cells and natural killer cells [3]. CCL2 was a 76-amino acid protein that was originally purified and cloned from human gliomas and myelomonocytic cells in 1989 [4]. CCL2 was the first chemokine discovered in the C-C subfamily of chemokines and was produced by a variety of cells. Some studies have implicated that CCL2 was an active participant in the tumor microenvironment $[5,6]$. CCL18 was a newly defined member of C-C subgroup of chemokines. It played an important role in inflammation and generation of the immune response. However, the involvement of CCL18 in cancer was not clear.

\section{Biomed Central}

(c) 2013 Wu et al.; licensee BioMed Central Ltd. This is an Open Access article distributed under the terms of the Creative Commons Attribution License (http://creativecommons.org/licenses/by/2.0), which permits unrestricted use, distribution, and reproduction in any medium, provided the original work is properly cited. 
Vascular endothelial growth factor (VEGF) was firstly isolated from the culture supernatant and the ascites of rodent tumors a potent vascular permeability factor [7]. It was thought to be one of the most important factors promoting vascularization [8]. It played a role in both physical and malignant conditions [9]. Previous studies reported that serum VEGF level was high in several cancers, such as breast cancer and colon cancer $[10,11]$.

The aim of this study was to investigate the relationship between the preoperative serum levels of CCL2, CCL18, and VEGF, and the clinicopathological factors in patients with gastric cancer.

\section{Methods}

\section{Study population}

From July 2005 to December 2005, 60 patients who underwent curative gastrectomy at department of Gastric Cancer and Soft Tissue Sarcoma in Shanghai Cancer Hospital were included into this study. All patients were confirmed as gastric cancer by preoperative biopsy. No patients received neoadjuvant treatment or preoperative blood transfusion. Additionally, 20 normal persons were designated as the control. Staging was performed according to the American Joint Committee on Cancer (AJCC) TNM Staging Classification for Carcinoma of the Stomach (6th edition, 2002). Data were retrieved from patients' operative and pathological reports. The written informed consent had been obtained from all the patients, and this study was approved by the Ethical Committee of Shanghai Cancer Center of Fudan University.

\section{Blood samples}

Blood samples were obtained by peripheral venous puncture on the day before operation. After clotting and within an hour of collection, the blood samples were centrifuged at $3000 \mathrm{~g}$ for $5 \mathrm{~min}$ and serum aliquots were stored at $-80^{\circ} \mathrm{C}$ until analysis.

\section{ELISA}

The measurement of CCL2, CCL18, and VEGF was performed using Quantikine human CCL2, CCL18, and VEGF sandwich enzyme-linked immunosorbent assay (ELISA) kits (Genzyme Corporation, USA) according to the manufacturer's instructions. All assays were duplicated.

\section{Statistical analysis}

Continuous variables and categorical variables were expressed as mean \pm 1 standard deviation and percentages, respectively. The Kolmogorov-Smirnov test was used to assess whether the continuous variables conformed to a Gaussian pattern. Comparisons of continuous variables between independent groups were performed with Student's unpaired $t$-test or Mann-Whitney non-parametric test. Comparisons of continuous variables between related groups were performed with Student's paired $t$-test and non-parametric Wilcoxon's signed-rank test. Linear correlations were assessed by calculating Pearson's correlation coefficient or the non-parametric Spearman's rho. The accepted level of significance was $P<0.05$. Statistical analyses and graphics were performed with the SPSS 13.0 statistical package (SPSS, Inc., Chicago, IL).

\section{Results}

\section{Clinicopathological characteristics}

There were 14 males and 6 females (2.33:1) with a mean age of 56 years in normal persons. There were 41 males and 19 females (2.16:1) with a mean age of 55 years. There was 7 (11.7\%) early gastric cancers and 53 (88.3\%) advanced gastric cancers. According to histological type, well-differentiated tumors were observed in $1(1.7 \%)$ patients, moderately-differentiated in $18(30.0 \%)$ patients, and poorly-differentiated tumors in remaining 41 (68.3\%) patients. Lymph node metastasis was observed in 40 patients, the metastasis rate was $66.7 \%$. The distribution of pathological stage was as follows: $11(18.3 \%)$ patients belonged to stage I, 9 (15.1\%) II, 23 (38.3\%) III, 17 (28.3\%) IV.

\section{Serum CCL12, CCL18, and VEGF values}

The serum values of CCL2, CCL18, and VEGF in normal persons were $15.95 \pm 1.15(\mathrm{pg} / \mathrm{ml}), 42.46 \pm 13.97(\mathrm{pg} / \mathrm{ml})$, and $59.02 \pm 6.28(\mathrm{pg} / \mathrm{ml})$, respectively. The serum values of CCL2, CCL18, and VEGF in gastric cancers were $25.05 \pm$ $1.22(\mathrm{pg} / \mathrm{ml}), 115.94 \pm 22.56(\mathrm{pg} / \mathrm{ml})$, and $132.92 \pm 10.50$ $(\mathrm{pg} / \mathrm{ml})$, respectively. The levels were significantly higher in patients than that of control groups (Table 1).

\section{Presence of malignancy}

Serum VEGF and CCL2 levels were significant independent predictors for the presence of gastric cancer. The optimal predictive model $\left(\chi^{2}=41.470, \mathrm{df}=3, \mathrm{~N}=80\right.$, Negelkerke $\left.R^{2}=0.599, P<0.001\right)$ predicted presence of

Table 1 Comparisons of serum CCL2, CCL18, and VEGF levels between gastric cancer patients and control groups

\begin{tabular}{lllll}
\hline & $\mathbf{N}$ & Mean $(\mathbf{p g} / \mathbf{m l})$ & Std. deviation & $\mathbf{P}$ \\
\hline CCL2 & & & & $<0.001$ \\
\hline Patients & 60 & 25.05 & 1.22 & \\
\hline Controls & 20 & 15.95 & 1.15 & $<0.001$ \\
\hline CCL18 & & & & \\
\hline Patients & 60 & 115.94 & 22.56 & $<0.001$ \\
\hline Controls & 20 & 42.46 & 13.97 & \\
\hline VEGF & & & & \\
\hline Patients & 60 & 132.92 & 10.50 & \\
\hline Controls & 20 & 59.02 & 6.28 & \\
\hline
\end{tabular}


malignancy with $93.3 \%$ sensitivity and $60.0 \%$ specificity. With a serum VEGF levels cut-off values $\geq 68.2 \mathrm{pg} / \mathrm{ml}$, the sensitivity and specificity of VEGF to distinguish patients from controls were $83 \%$ and $60 \%$, respectively. In contrast, with a serum CCL2 levels cut-off values $\geq 16.6 \mathrm{pg} / \mathrm{ml}$, the sensitivity and specificity of CCL2 to distinguish patients from controls were $78 \%$ and $60 \%$, respectively.

With a cut-off value of $\geq 1272.8$, the VEGF*CCL2 predicted the presence of gastric cancer with $83 \%$ sensitivity and $80 \%$ specificity (Table 2 ). The concentrations product yielded the largest area under the ROC curve $(0.895$, $P<0.001$ ) (Figure 1).

\section{Correlation of preoperative serum markers and clinicopathological features}

Preoperative serum CCL2 was significantly correlated to age $(\mathrm{r}=0.433, P=0.001)$ and $\mathrm{N}$ stage $(\mathrm{r}=-0.266$, $P=0.040$ ). Preoperative serum CCL18 was significantly associated with histology type $(\mathrm{r}=-0.296, P=0.022), \mathrm{N}$ stage $(\mathrm{r}=-0.384, P=0.002)$, and TNM stage $(\mathrm{r}=-0.386$, $P=0.002)$. Preoperative serum VEGF was significantly correlated to T stage $(\mathrm{r}=0.598, P=0.000)$ and $\mathrm{N}$ stage $(\mathrm{r}=0.312$, $P=0.015)$, and TNM stage $(\mathrm{r}=0.531, P=0.000)$. There were no statistically significant correlation of CCL2, CCL18, and VEGF to gender, vascular invasion, and nervous invasion (Table 3).

\section{Discussion}

Poor survival of gastric cancer was largely due to late-stage diagnosis. Late-stage diagnosis can be attributed to the fact that the disease was relatively "asymptomatic" in its early stage and nonspecific complaints in its late-stage. Therefore, it was necessary to find out some markers for early diagnosis of gastric cancer. Thus, we measured the concentration of CCL2, CCL18, and VEGF in a series of 60 serum samples from gastric cancer. Additionally, a series of 20 serum samples from healthy people was selected as controls. Moreover, we investigated the relationship between

Table 2 Cut-off values, sensitivity and specificity of VEGF*CCL2 for distinguishing gastric cancer patients from controls

\begin{tabular}{lll}
\hline Cut-off/upper than or equal (+) & Sensitivity (\%) & Specificity (\%) \\
\hline VEGF* ${ }^{*} C L 2$ & & \\
\hline 1205.6 & 85 & 70 \\
\hline 1212.6 & 85 & 75 \\
\hline 1242.6 & 83 & 75 \\
\hline $\mathbf{1 2 7 2 . 8}$ & $\mathbf{8 3}$ & $\mathbf{8 0}$ \\
\hline 1291.4 & 82 & 80 \\
\hline 1332.3 & 80 & 80 \\
\hline
\end{tabular}

In bold, the reference cut-off value that combines optimal sensitivity and specificity for clinical use.

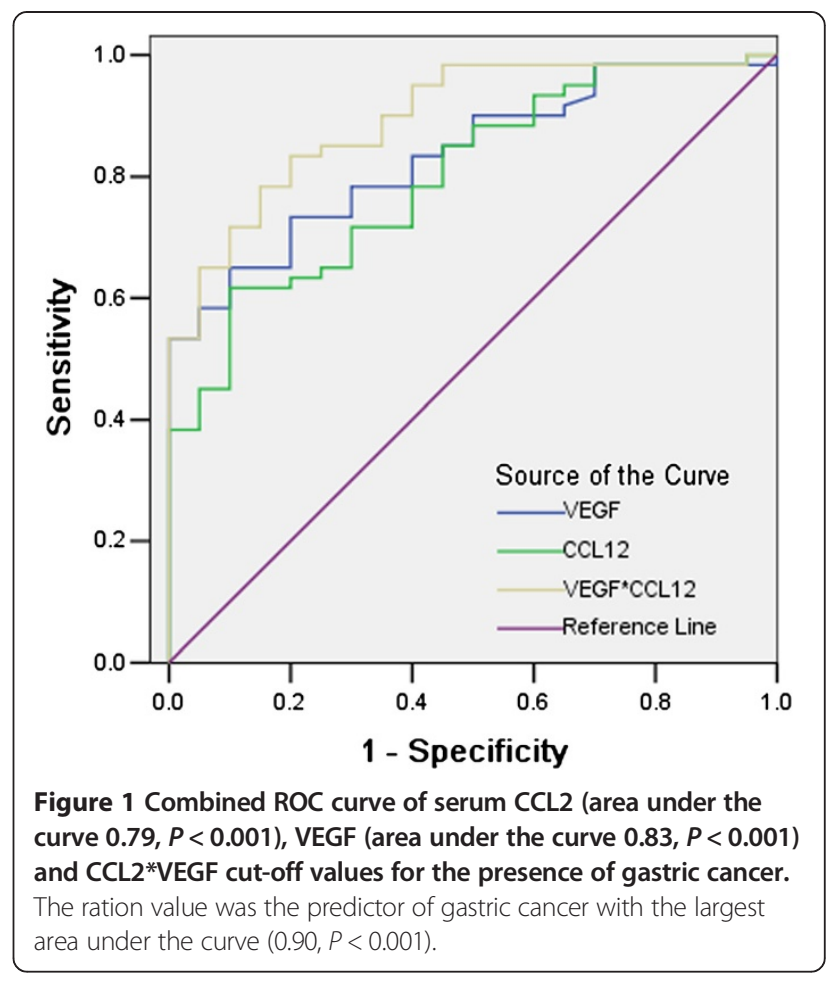

the levels of these markers and the clinicopathological factors. To our knowledge, no published studies have simultaneously studied the diagnostic value of serum CCL2, CCL18, and VEGF in gastric cancer patients.

In the current study, we found that the serum levels of CCL2, CCL18, and VEGF markedly increased in gastric cancer group compared to control group. It was generally accepted that CCL2 was a potent proinflammatory mediator produced by several cells including monocytes [12]. Additionally, tumor cells or peritumoral components can produce cytokines [13]. CCL2 has been

Table 3 The relation between preoperative serum VEGF, CCL12, CCL18 and clinicopathological parameters in gastric cancer patients

\begin{tabular}{|c|c|c|c|c|c|c|}
\hline \multirow[t]{2}{*}{ Parameters } & \multicolumn{2}{|l|}{ VEGF } & \multicolumn{2}{|l|}{$\mathrm{CCL} 2$} & \multicolumn{2}{|l|}{ CCL18 } \\
\hline & $r$ & $P$ & $r$ & $P$ & $r$ & $P$ \\
\hline$\overline{\mathrm{T}}$ & 0.598 & 0.000 & -0.065 & 0.623 & -0.092 & 0.485 \\
\hline $\mathrm{N}$ & 0.312 & 0.015 & -0.266 & 0.040 & -0.384 & 0.002 \\
\hline$M$ & 0.166 & 0.204 & 0.046 & 0.724 & -0.220 & 0.091 \\
\hline$\overline{T N M}$ & 0.531 & 0.000 & -0.118 & 0.370 & -0.386 & 0.002 \\
\hline Histological type & -0.115 & 0.382 & 0.164 & 0.211 & -0.296 & 0.022 \\
\hline Venous invasion & -0.032 & 0.807 & 0.089 & 0.499 & -0.112 & 0.393 \\
\hline Neuron invasion & 0.061 & 0.644 & -0.006 & 0.962 & -0.025 & 0.848 \\
\hline Gender & 0.029 & 0.826 & 0.125 & 0.341 & 0.088 & 0.504 \\
\hline Age & 0.123 & 0.348 & 0.433 & 0.001 & -0.084 & 0.525 \\
\hline
\end{tabular}

$r$ Correlation coefficients $(r)$. 
investigated in serum of patients with some tumors like breast cancer, ovarian tumor, and gastric cancer [13-15]. Tonouchi et al. [15] reported that the serum concentration of CCL2 in patients with carcinoma was significantly lower than that in controls, which was not consistent with the current study. In this study, we found that the serum levels of CCL2 markedly increased in gastric cancer group compared to control group. The exact mechanism resulting in this contradiction was not clear. It was possible that the decreased serum level of CCL2 may reflect increased local consumption in tumor [15]. However, others have shown statistically significant increases in serum CCL2 levels in patients compared with healthy controls $[13,14]$. CCL18 played a role in the homing of $\mathrm{T}$ and $\mathrm{B}$ cells participating in the immune response. It was reported that CCL18 was correlated to the autoimmune disease. However, the reports about $\mathrm{CC} 18$ in cancer were rare. In this study, we found that CCL18 markedly increased in gastric cancer group compared to control group. However, it was not independent predictor for presence of gastric cancer. It was well known that vascular endothelial growth factor (VEGF) family played an important role in vasculogenesis and angiogenesis. Among the VEGF family members, VEGFA played an essential role in angiogenesis. However, the significant value of serum levels of other VEGF members (VEGF-C and VEGF-d) has been reported [16-18]. However, previous studies on serum VEGF-C and VEGF-D in gastric cancer patients have presented with contradictory results. Wang et al. [16] reported that higher serum levels significantly associated with the presence of malignancy and lymph node metastasis. To the contrary, AI-Moundhri et al. [17] did not showed significant differences of serum VEGF-C between patients and control groups, and higher VEGF-D in patients. In review of inconsistent reports, we conducted logistic regression analysis, and found that serum VEGF was an independent predictor of the presence of gastric cancer. Furthermore, we performed the ROC curve to investigate the predictive value of gastric cancer, and results showed that VEGF"CCL2 could provide higher accuracy for distinguishing gastric cancer patients from controls than alone VEGF or CCL.

The correlation of CCL2, CCL18, and VEGF with clinicopathological variables has been elucidated in various tumors. Tonouchi $\mathrm{H}$ et al. reported that the concentration of serum CCL2 in gastric cancer patients decreased in accordance with disease progression [15]. Zohny SF et al. reported that serum CCL18 was significantly increased in epithelial ovarian cancer with early stages compared to those with late stages [19]. In this study, we found the similar results. It was suggested that the consumption of CCL2 and CCL18 increased and production decreased in accordance with disease progression.

\section{Conclusions}

CCL2 played an important role in immune response to tumor. Together with VEGF, they were useful biomarkers in predicting gastric cancer.

\section{Competing interests}

The authors declare that they have no competing interests.

\section{Authors' contributions}

Conceived and designed the experiments: JW XL YW. Performed the experiments: JW XL. Analyzed the data: JW XL YW. Wrote the paper: JW XL. All authors read and approved the final manuscript.

\section{Acknowledgments}

We thank Hong Cai for revising the manuscript.

Received: 30 November 2012 Accepted: 16 May 2013

Published: 22 May 2013

\section{References}

1. Shibata A, Parsonnet J: Stomach cancer. In Cancer epidemiology and prevention. 3rd edition. Edited by Schottenfeld D, Fraumeni JF. New York: Oxford University Press; 2006:707-720.

2. Parkin DM, Bray F, Ferlay J, Pisani P: Global cancer statistics, 2002. CA Cancer J Clin 2005, 55:74-108.

3. Singh S, Sadanandam A, Singh PK: Chemokines in tumor angiogenesis and metastasis. Cancer Metastasis Rev 2007, 26:453-467.

4. Leonard EJ, Yoshimura T: Human monocyte chemoattractant protein-1 (MCP-1). Immunol Today 1990, 11:97-101.

5. Salcedo R, Ponce ML, Young HA, Wasserman K, Ward JM, Kleinman HK, Oppenheim JJ, Murphy WJ: Human endothelial cells express CCR2 and respond to $\mathrm{MCP}-1$ : direct role of $\mathrm{MCP}-1$ in angiogenesis and tumor progression. Blood 2000, 96:34-40.

6. Saji H, Koike M, Yamori T, Saji S, Seiki M, Matsushima K, Toi M: Significant correlation of monocyte chemoattractant protein-1 expression with neovascularisation and progression of breast carcinoma. Cancer 2001, 92:1085-1091.

7. Senger DR, Galli SJ, Dvorak AM, Perruzzi CA, Harvey VS, Dvorak HF: Tumor cells secrete a vascular permeability factor that promotes accumulation of ascites fluid. Science 1983, 219:983-985.

8. Kim KJ, Li B, Winer J, Armanini M, Gillett N, Phillips HS, Ferrara N: Inhibition of vascular endothelial growth factor-induced angiogenesis suppresses tumour growth in vivo. Nature 1993, 362:841-844.

9. Ferrara N, Gerber HP, LeCouter J: The biology of VEGF and its receptors. Nat Med 2003, 9:669-676.

10. Nishimura R, Nagao K, Miyayama H, Matsuda M, Baba K, Yamashita H, Fukuda M: Higher plasma vascular endothelial growth factor levels correlate with menopause, overexpression of $\mathrm{p} 53$, and recurrence of breast cancer. Breast Cancer 2003, 10:120-128.

11. De Vita F, Orditura M, Lieto E, Infusino S, Morgillo F, Martinelli E, Castellano P, Romano C, Ciardiello F, Catalano G, Pignatelli C, Galizia G: Elevated perioperative serum vascular endothelial growth factor levels in patients with colon carcinoma. Cancer 2004, 100:270-278.

12. Gmyrek GB, Sozanski R, Jerzak M, Chrobak A, Wickiewicz D, Skupnik A, Sieradzka U, Fortuna W, Gabrys M, Chelmonska-Soyta A: Evaluation of monocyte chemotactic protein-1 levels in peripheral blood of infertile women with endometriosis. Eur J Obstet Gynecol Reprod Biol 2005, 122:199-205.

13. Hefler L, Tempfer C, Heinze G, Mayerhofer K, Breitenecker G, Leodolter S, Reinthaller A, Kainz C: Monocyte chemoattractant protein-1 serum levels in ovarian cancer patients. Br J Cancer 1999, 81:855-859.

14. Dehqanzada ZA, Storrer CE, Hueman MT, Foley RJ, Harris KA, Jama YH, Kao TC, Shriver CD, Ponniah S, Peoples GE: Correlations between serum monocyte chemotactic protein-1 levels, clinical prognostic factors, and HER-2/neu vaccine-related immunity in breast cancer patients. Clin Cancer Res 2006, 12(2):478-486.

15. Tonouchi H, Miki C, Tanaka K, Kusunoki M: Profile of monocyte chemoattractant protein-1 circulating levels in gastric cancer patients. Scand J Gastroenterol 2002, 37:830-833. 
16. Wang TB, Deng MH, Qiu WS, Dong WG: Association of serum vascular endothelial growth factor-C and lymphatic vessel density with lymph node metastasis and prognosis of patients with gastric cancer. World J Gastroenterol 2007, 13:1794-1798.

17. Al-Moundhri MS, Al-Shukaili A, Al-Nabhani M, Al-Bahrani B, Burney IA, Rizivi $A$, Ganguly SS: Measurement of circulating levels of VEGF-A, $-C$, and -D and their receptors, VEGFR-1 and -2 in gastric adenocarcinoma. World J Gastroenterol 2008, 14:3879-3883.

18. Tsirlis TD, Kostakis A, Papastratis G, Masselou K, Vlachos I, Papachristodoulou A, Nikiteas NI: Predictive significance of preoperative serum VEGF-C and VEGF-D, independently and combined with CA19-9, for the presence of malignancy and lymph node metastasis in patients with gastric cancer. J Surg Oncol 2010, 102:699-703.

19. Zohny SF, Fayed ST: Clinical utility of circulating matrix metalloproteinase-7 (MMP-7), CC chemokine ligand 18 (CCL18) and CC chemokine ligand 11 (CCL11) as markers for diagnosis of epithelial ovarian cancer. Med Oncol 2010, 27(4):1246-1253.

doi:10.1186/1472-6890-13-15

Cite this article as: Wu et al:: Predictive value of preoperative serum CCL2, CCL18, and VEGF for the patients with gastric cancer. BMC Clinical Pathology 2013 13:15.

\section{Submit your next manuscript to BioMed Central and take full advantage of:}

- Convenient online submission

- Thorough peer review

- No space constraints or color figure charges

- Immediate publication on acceptance

- Inclusion in PubMed, CAS, Scopus and Google Scholar

- Research which is freely available for redistribution 